\title{
BMJ Open Designing and pilot testing of a leadership intervention to improve quality and safety in nursing homes and home care (the SAFE-LEAD intervention)
}

Terese Johannessen, ${ }^{1}$ Eline Ree, ${ }^{1}$ Torunn Strømme, ${ }^{1}$ Ingunn Aase, ${ }^{1}$ Roland Bal, ${ }^{2}$ Siri Wiig ${ }^{1}$

To cite: Johannessen T, Ree E, Strømme T, et al. Designing and pilot testing of a leadership intervention to improve quality and safety in nursing homes and home care (the SAFELEAD intervention). BMJ Open 2019;9:e027790. doi:10.1136/ bmjopen-2018-027790

- Prepublication history for this paper is available online. To view these files, please visit the journal online (http://dx.doi org/10.1136/bmjopen-2018027790).

Received 7 November 2018 Revised 6 May 2019 Accepted 20 May 2019

\section{Check for updates}

(c) Author(s) (or their employer(s)) 2019. Re-use permitted under CC BY-NC. No commercial re-use. See rights and permissions. Published by BMJ.

${ }^{1}$ Faculty of Health Sciences, SHARE - Centre for Resilience in Healthcare, University of Stavanger, Stavanger, Norway ${ }^{2}$ School of Health Policy \& Management, Erasmus University, Rotterdam, The Netherlands

Correspondence to Ms Terese Johannessen; terese.johannessen@uis.no

\section{ABSTRACT}

Objective To describe the design of a leadership intervention for nursing home and home care, including a leadership guide for managers to use in their quality and safety improvement work. The paper reports results from the pilot test of the intervention and describes the final intervention programme.

Design Qualitative design, using the participation of stakeholders.

Methods The leadership guide and intervention were designed in collaboration with researchers, coresearchers and managers in nursing homes and home care organisations, through workshops and focus group interviews. The pilot test consisted of three workshops with managers working on the leadership guide, facilitated and observed by researchers, and evaluated by means of observation and focus group interviews with the participants. The analysis combined the integration of data from interviews and observations with directed content analysis.

Setting Norwegian nursing homes and home care services.

Participants Managers at different levels in three nursing homes and two home care services, coresearchers, and patient and next-of-kin representatives.

Results The managers and coresearchers suggested some revisions to the leadership guide, such as making it shorter, and tailoring the terminology to their setting. Based on their suggestions, we modified the intervention and developed learning resources, such as videos demonstrating the practical use of the guide. Evaluation of the pilot test study showed that all managers supported the use of the guide. They adapted the guide to their organisational needs, but found it difficult to involve patients in the intervention.

Conclusions A participatory approach with stakeholders is useful in designing a leadership intervention to improve quality and safety in nursing homes and home care, although patient participation in its implementation remains difficult. The participatory approach made it easier for managers to adapt the intervention to their context and to everyday quality and safety work practice.
Strengths and limitations of this study

- The main strength of this study is that the intervention design drew on input from researchers, coresearchers, future users of the Improving Quality and Safety in Primary Care-Implementing a Leadership Intervention in Nursing Homes and Home care intervention and patient and next-of-kin representatives, and several data sources.

- The sample of managers and units in the pilot test is limited. However, the feedback received during interviews and pilot testing of the intervention was consistent across the sample, and supported by the feedback received from the coresearchers.

- The intervention design needs to be made sustainable over time without researcher involvement, and patients should be more actively involved in its implementation.

\section{BACKGROUND}

Translating research-based findings into practice in healthcare is difficult. ${ }^{1-4}$ Improvement interventions too often fail, and management teams have to know how to implement evidence-based knowledge to facilitate quality improvement at the local service level. ${ }^{56}$ Quality improvement interventions may have multiple components designed to enable knowledge translation to foster change in the behaviour of people and organisational systems, and can target any or all of the quality domains (safety, effectiveness, efficiency, timeliness, equity and patient-centredness) where performance is unsatisfactory. ${ }^{7}$

The recent literature on intervention design emphasises the importance of using theory, acknowledging interventions as both social and technical, and the role of context. ${ }^{6}$ Perhaps the most important need highlighted in the literature is for a more 
active and collaborative design of the intervention, involving stakeholders such as service users, practitioners and researchers aiming to solve practical problems in a way that emphasises shared reflection and collective inquiry. ${ }^{368}$ More effort is needed to design an intervention that incorporates all of those elements. ${ }^{6}$

Leadership is central to a healthcare organisation's effort to improve quality and safety. ${ }^{9-14}$ A recent systematic review shows how disengaged leadership, leadership turnover, poor organisational culture and dysfunctional external relations are characteristic of healthcare organisations that are struggling with quality and safety. ${ }^{15}$ However, our knowledge of how best to design interventions to support healthcare managers with new tools and competence to diagnose and improve their organisational quality and safety challenges needs to be strengthened. ${ }^{12} 16$ In the Norwegian context, policy-makers have called for the development of stronger competence among managers in relation to quality and safety improvement, in addition to the leadership skills and tools to translate research-based knowledge into practice. Policy-makers and research funding bodies have paid special attention to the primary care setting in terms of nursing home and home care managers who need the tools and competence to achieve the goals of sound professional practice, reduced patient harm and improved service quality. ${ }^{17} 18$ Our study contributes to fill this knowledge and tool gap by designing a leadership intervention for nursing home and home care managers.

\section{The Improving Quality and Safety in Primary Care- Implementing a Leadership Intervention in Nursing Homes and Home care study}

The intervention described and pilot tested in this paper is part of a larger study titled 'Improving Quality and Safety in Primary Care-Implementing a Leadership Intervention in Nursing Homes and Home care' (SAFELEAD).${ }^{16}$ The SAFE-LEAD study builds on the European Union seventh Framework Program funded project 'Quality and Safety in Europe by Research' (QUASER) conducted in hospitals, by applying research findings to Norwegian nursing homes and home care settings. The QUASER guide defines quality care as care that is clinically effective, safe and patient centred. Care coordination is central in the understanding of quality in the Norwegian primary care context and is incorporated into the SAFE-LEAD study's conceptualisation of quality. The original QUASER guide ${ }^{19}$ is theoretically anchored in the Organising for Quality framework ${ }^{20}$ and structured around eight common quality challenges: structure, politics, culture, education, emotions, technical and physical conditions, leadership, and external demands. ${ }^{19}$ It consists of a series of questions to stimulate reflection and an accompanying decision-aid menu of potential options, including empirical examples of possible quality and safety improvement solutions across macro, meso and micro system levels. The guide is designed to facilitate patient safety and quality improvement in clinical practice and service delivery, by giving managers a systematic way to identify the strengths and weaknesses of their improvement approach and reflect on what is required to develop quality improvement and patient safety efforts tailored to their needs. ${ }^{12} 131920$

Based on the original QUASER-guide, the SAFE-LEAD study will develop, implement and evaluate a researchbased leadership guide for the nursing home and home care context in Norway. This paper focuses on the design and modification of the leadership guide, and the form and content of a pilot intervention programme. ${ }^{16}$

\section{Aim and research question}

The aims of this paper were (1) to describe the design of the leadership intervention; (2) to conduct a pilot test of the intervention to ascertain the feasibility of the intervention design and receive input for modifications and (3) to describe the final intervention programme prior to implementation. The objectives of the pilot test were to receive information on the functionality of the leadership guide, the pedagogical approaches, and the structure and content of the intervention. Testing outcome measures was not an objective. ${ }^{16}$

The following research question was addressed: How can a leadership intervention for improving quality and safety be designed for implementation in nursing homes and home care contexts?

\section{METHODS}

This SAFE-LEAD study has a mixed-methods design; this paper uses a combination of qualitative methods. To guide the design of the SAFE-LEAD intervention, we used the Medical Research Council's (MRC) guidance framework for the development and evaluation of complex interventions. ${ }^{21}$ The MRC's guidance views healthcare interventions as flexible, non-linear processes, giving equal attention to all phases (development, testing, evaluation and wider application). Also noteworthy is that while advocating the systematic development of interventions, it stresses the importance of context in implementation and allows for adaptation of an intervention to the local setting to ensure its success in practice. ${ }^{21}$

The Knowledge to Action framework ${ }^{2}$ guided the development process. According to this framework, translating the leadership guide into practice requires an organisation to identify the problems it needs to solve; tailor the guide to its own settings and contexts; assess and address barriers to its use; implement the intervention; monitor the implementation and evaluate the outcomes. ${ }^{22}$ One part of the development process was the identification of contextual factors in the Norwegian nursing home and home care setting that can affect outcomes. These results will be published in a separate paper.

\section{Data collection}

In the following, we describe the data collection methods in the different phases of the development and pilot testing, the data sources (table 1), and the data analysis. 


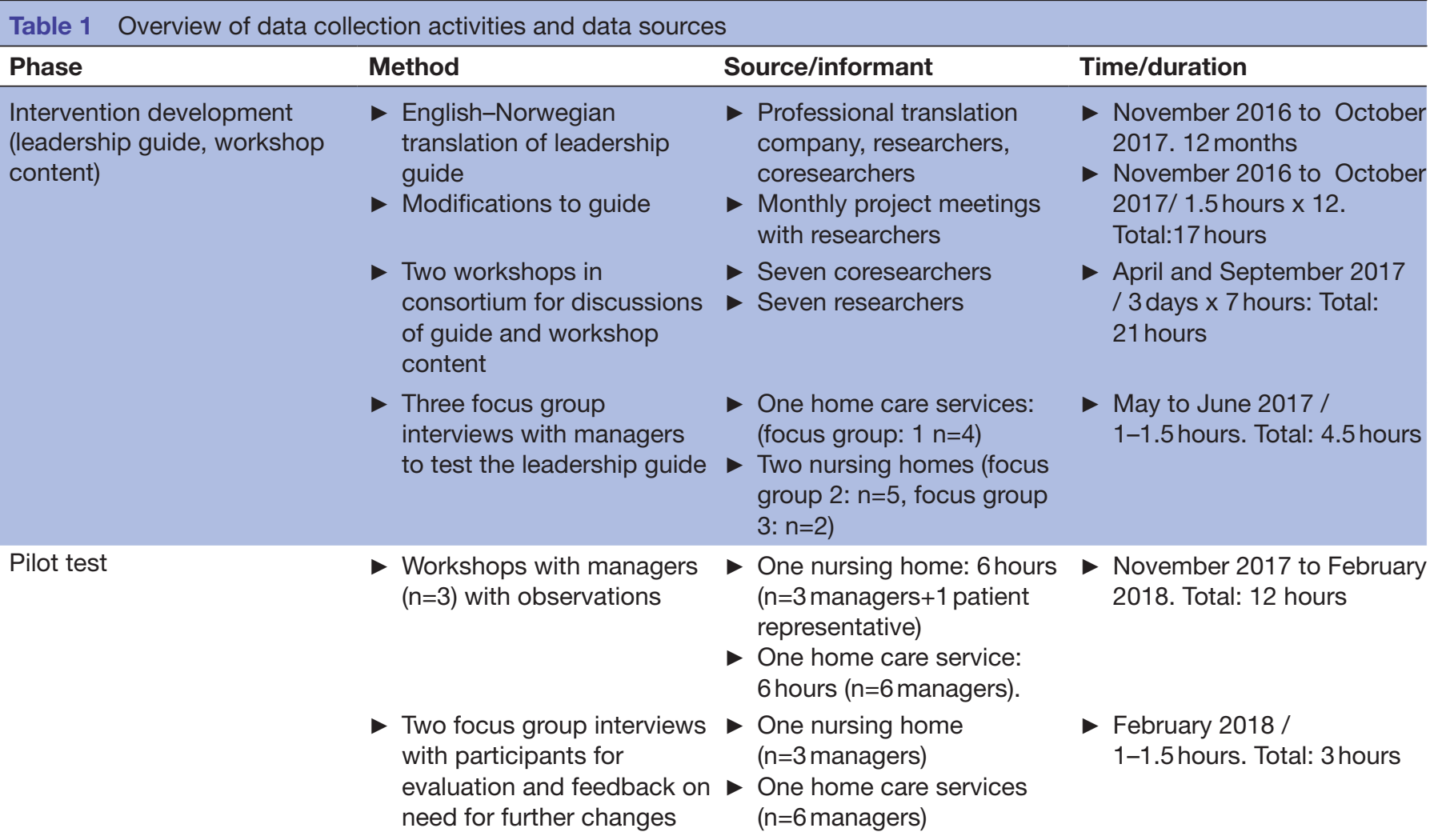

\section{Intervention development}

The design of the intervention planned to include a 6-month intervention consisting of 3-4 workshops with manager teams working with the leadership guide. The workshops were scheduled in advance, ${ }^{16}$ but their content and structure, as well as the leadership guide, were developed in a participatory process. ${ }^{3}{ }^{23}$ First, the QUASER Hospital Guide was translated from English to Norwegian by a professional translation service. Further language adjustments were conducted in collaboration with the project team (researchers with backgrounds in nursing, health psychology, safety science, engineering and health management), coresearchers in the project (nurse counsellors from different municipalities, patient and nextof-kin representatives) and future users (managers from nursing homes and home care). An important part of the development process was to adapt the leadership guide to the Norwegian nursing home and home care setting. The original QUASER guide is based on empirical findings from European hospitals. The SAFE-LEAD project developed a version that was adapted to Norwegian nursing homes and home care settings. The development process lasted from November 2016 to November 2017. There were several iterations before agreement was reached on the final version. This process comprised meetings with the project team and two workshops with coresearchers to obtain feedback on language, format and content. These arenas were also used to discuss the structure and content of the intervention workshops.

The leadership guide was refined based on coresearchers' feedback, and in May 2017, we conducted three focus group interviews with potential users of the guide. The participants were managers, development nurses and nurses from two nursing homes $(\mathrm{n}=7)$ and one home care service $(n=4)$, to test and receive feedback on the leadership guide. The focus group lasted for 60-90 min (table 1). The participants were given time to read the leadership guide and the interview guide beforehand. The interview guide contained questions about the content, structure and format of the leadership guide, in addition to what the managers considered important when using the guide in their daily quality and safety work (eg, amount and type of researcher involvement and facilitation, and training needs). The purpose of the focus group interviews was to elicit the participants' thoughts about the clarity, usefulness and content of the leadership guide, and to identify learning support needs so that the guide could be used for quality improvement work. Based on the theory, literature, input from coresearchers and potential users, we designed the SAFELEAD intervention.

\section{Pilot test}

The pilot test evaluated the feasibility of the intervention. We conducted a process evaluation of the pilot test according to the study protocol ${ }^{16}$ focusing on guide functionality, pedagogical approach and intervention content, in order to asses needs for modifications. ${ }^{24}$

The SAFE-LEAD intervention was pilot tested for feasibility in two organisations located in the same urban Norwegian municipality. The pilot test lasted 2-3 months (table 1). One coresearcher from the Centre 
for Development of Institutional and Home Care services (USHT) in the municipality recruited the study sites. All invited participants consented to participate. No one who had been invited declined. The organisations were one large nursing home and one large home care service. The same home care service site also participated in the focus group interviews in the development phase of the leadership guide, to assess if its suggested changes had improved the guide. One management team in each organisation participated in the workshops, which were conducted at their own workplace. The management team of the nursing home consisted of three managers and one patient representative. The management team from the home care services consisted of six managers. Each organisation held three workshops. During the workshops, one researcher facilitated discussion and one or two researchers observed and took notes. In addition to taking observational notes during the workshops, we conducted semistructured focus group interviews with the participants in both facilities after the final workshop to evaluate their experiences with the leadership guide, and with the intervention workshops during the pilot test period. The interview guide contained questions about (1) experiences with the leadership guide (eg, content, language, usefulness), (2) the workshop (eg, content, structure, usefulness, challenges, material received in advanced, web tool) and (3) experiences with their work processes in between workshops. We structured the workshop agendas around the three-step process in the leadership guide (figure 1). During all workshops, the researchers observed and took notes according to a guide based on the workshop agendas (eg, reflections concerning quality and safety work, challenges and goals, as well as work processes and progress).

\section{Data analysis}

The data analysis was inspired by Strøm and Fagermoen's ${ }^{25}$ approach to integrating data from interviews and observation collected during a year-long process of collaborative development. The key component of this strategy is the interweaving of observation and interview data derived from sequences of interactive situations, such as the intervention design process, and analysing them as a complete body of material. The data material was analysed before integration. In our study, all focus group interviews were tape-recorded and transcribed verbatim. The focus group data were all subjected to directed content analysis ${ }^{26}$ according to the predefined categories of the leadership guide,${ }^{20}$ and according to training needs, and user experiences from the pilot test to enable evaluation of the intervention feasibility. ${ }^{27}$ The field notes were transcribed and analysed in a more descriptive manner ${ }^{28}$ that focused on sorting the contents of the field notes recorded during and after the consortium workshops and intervention workshops. The field notes were also analysed according to the functionality of the guide content in the development process, and according to intervention content and functionality during the pilot test. The aim of our integrative analysis was to produce a systematic, descriptive overview of discussions and decisions regarding the intervention development and content, the identified training needs and chronologically describe the results from the pilot test. In this way, we integrated the data sources collected over a 1-year period and described the process underlying the final intervention programme ready for implementation. All researchers and coresearchers collaborated in the process and during the data analysis, the preliminary

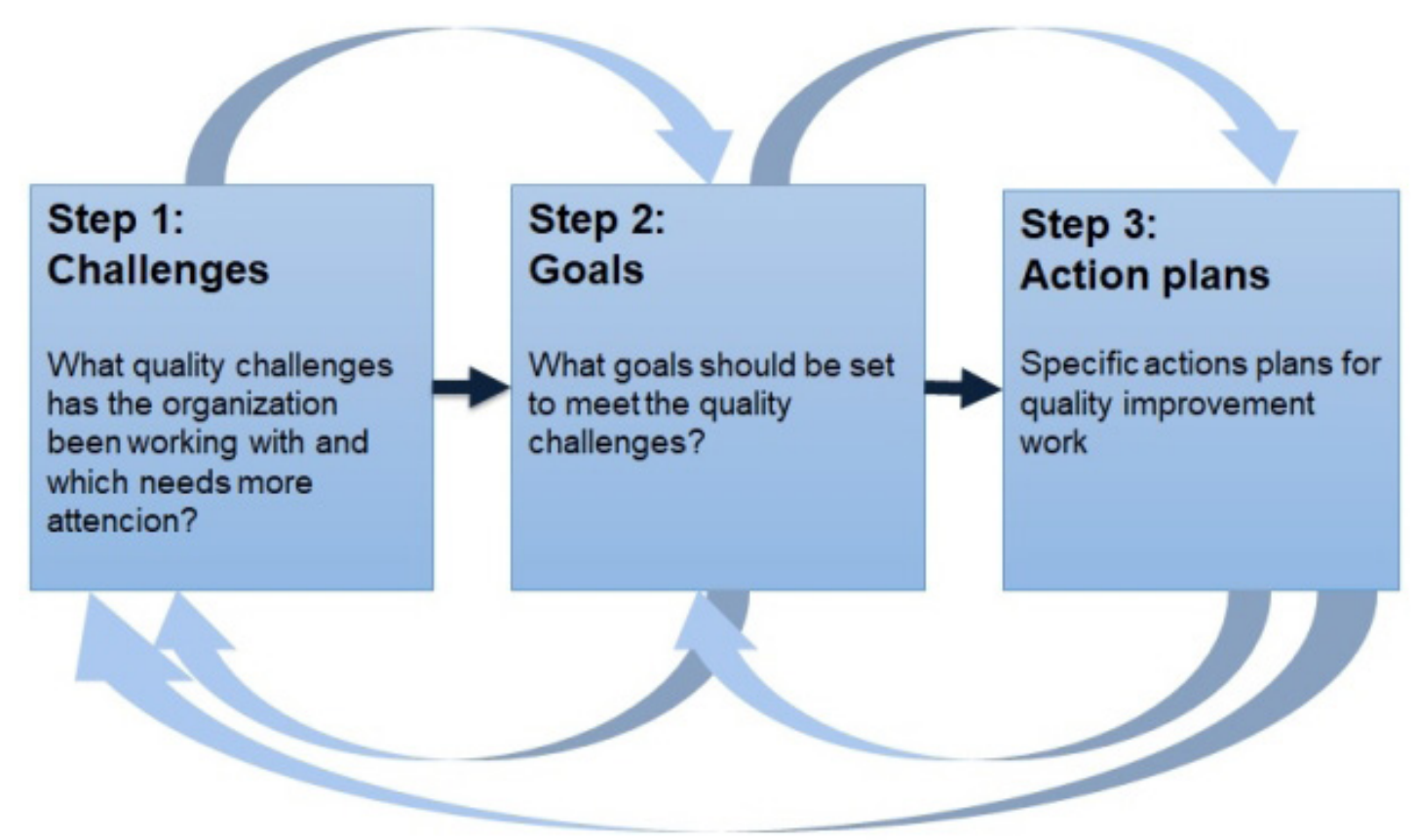

Figure 1 Three-step process. 
research findings were discussed in consortium meetings to ensure trustworthiness.

\section{Patient and public involvement statement}

The user, patient and next-of-kin perspectives are key throughout the SAFE-LEAD project from project development, design, data collection, analysis, through the dissemination of results. ${ }^{16}$ The development process has consisted of workshops with the co-researchers in the SAFE-LEAD consortium ensuring sound user involvement with perspectives from patient representatives, next-of-kin representatives and Patient and User Ombudsman and perspectives from future users of the guide (managers in nursing home and home care services). Three nurse-counsellors from the USHT were recruited by their municipality which is a partner in the project. These three nurse-counsellors are employed as coresearchers, in addition to one head of a USHT who was not paid, but who participated in semiannual project meetings. In addition, one patient representative and one next-of-kin representative were recruited as coresearchers in the SAFE-LEAD project after startup, by the project manager. The Patient and User Ombudsman was recruited as partner in the project and was involved in the project development and application for funding. The Patient and User Ombudsman has an important role in the stakeholder network and in quality assurance of the intervention design and pilot testing. The recruitment of participants and study sites was conducted in collaboration with the three USHT coresearchers. Managers from nursing home and home care participated in the development of the leadership guide, intervention workshop content and pilot test. One patient from the nursing home study site participated in the pilot test.

\section{RESULTS}

We now describe the intervention design process and the participatory approach. The results from the guide and workshops development process are presented, followed by results from the pilot test and the final adjusted intervention programme ready for full implementation in the SAFE-LEAD study. ${ }^{16}$

\section{Phase 1: development of leadership guide and workshop content \\ Development of leadership guide}

The leadership guide is built around a three-step process (figure 1) in which the organisations assess themselves on seven common quality challenges (figure 2). As illustrated in figure 2, both 'patient' (for nursing homes) and 'user' (for home care) are represented in the leadership guide.

The participants in the focus groups had several suggestions for the development of the leadership guide, which were in line with the input received from coresearchers
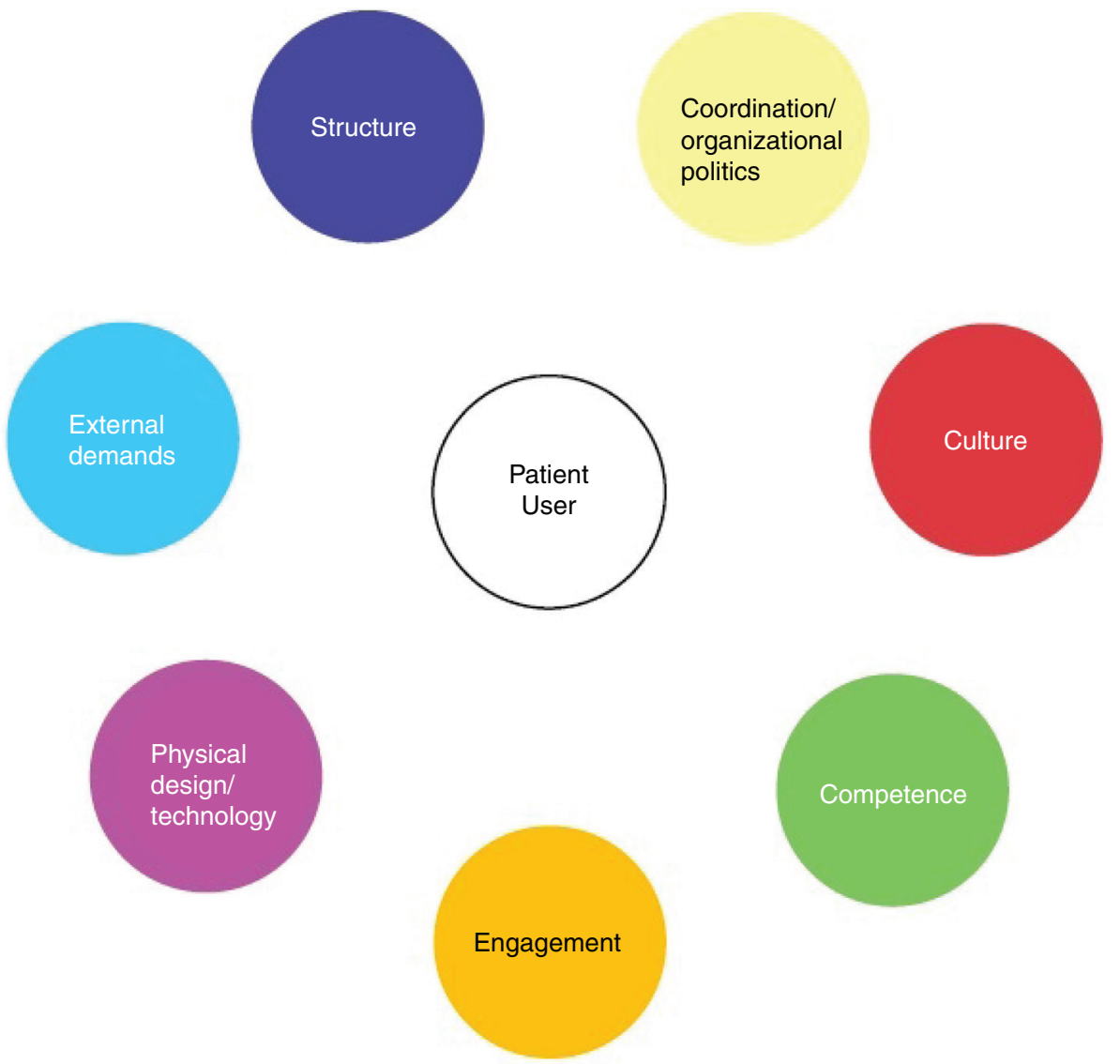

Figure 2 The seven challenges of quality improvement based on Bate et al. ${ }^{20}$ 
Table 2 Guide elements changed in the development process

Guide element from the QUASER guide

Structure

in the project. Their suggested revisions were related to the structure, language and content of the guide. Table 2 summarises the feedback. The translation of the guide was an iterative working process. Coresearchers and users of the guide reported having difficulties with some of the language after translation because it did not fit the vocabulary used in everyday practice. Thus, language revisions were made as a part of the development process and modification of the guide to fit the nursing home and home care settings.

The managers and coresearchers concurred that the leadership guide should be shorter, more detailed and easier to read. Several changes were made to condense the guide. The original version included approximately 80 pages of examples of quality improvement work in European hospitals. These examples were not considered especially relevant for the nursing home and home care settings and were therefore removed. Removing duplications and condensing several paragraphs and definitions also shortened the guide.
Some of the original eight quality challenges did not fit the Norwegian context and vocabulary when translated from English, resulting in a rewording of some of the challenges and expansion or restriction of some of the definitions. For example, the participants in the focus group and the coresearchers argued that since care coordination is a key domain of the Norwegian quality and safety work and reforms on policy level, it merited inclusion as a quality challenge. The 'political challenge' was, therefore, changed to 'care coordination and organisational politics.' Furthermore, the 'educational challenge' was reworded to 'competence,' and the 'emotional challenge' to 'engagement.' The 'leadership challenge,' one of the eight challenges in the original QUASER-guide, was removed, because the managers and coresearchers viewed leadership as inseparable from the other challenges. An important issue in the feedback on the guide content was that the patient/user was lacking in the guide and in figures used for visualisation. Consequently, the patient/user received more attention in the leadership guide, in definitions and goals, and in the visual presentation of the seven challenges by being placed in the centre, symbolising that quality work improves service quality for the patients/users (figure 2).

The participants supported our idea of developing a web version of the guide in addition to the paper version. The participants believed that a web version could provide simple, digital and visual presentation of the work in the leadership guide. They added that a web version would be easier to use and could be readily updated without losing earlier versions. Based on the feedback, we developed a digital interactive version of the leadership guide with the same content as the paper version. The digital web version allows for data storage and visualisation of the guide and evaluation over time.

\section{Development of workshop content}

The coresearchers and managers who participated in the focus groups offered the following suggestions about the content of the intervention workshops:

- Introduction of the leadership guide by a researcher in the first workshop.

- Specific homework and preparation between workshops.

- Learning tools to ease the managers' workload with the leadership guide (presentation of the guide and sample videos of the guide being used in practice).

It was suggested that the first workshop should start with one of the researchers introducing the leadership guide, to improve participants' understanding of its purpose, and to encourage its use in quality work. They also wanted short videos demonstrating practical examples of the guide in use. Based on the training needs identified in the focus group interviews, we developed four videos as learning resources. The first is a $10 \mathrm{~min}$ studio lecture presentation of the leadership guide. One of the researchers introduces the guide. The three other videos, each lasting about $6 \mathrm{~min}$, use actors to demonstrate the 
practical use of the guide. In each video, three actors portray managers from the home care service setting as they follow the three steps of the guide (figure 1). The videos cover the topics that were discussed in each of the first three workshops (assessment of quality challenges, setting goals, make action plans). All learning resources were emailed to the managers 1 week before the workshops and were available on the internet. The design of the video manuscripts was based on data and input from coresearchers' experiences with the home care services, and on focus group interviews with managers who responded to the draft version of the leadership guide.

Based on the input from the coresearchers and the focus group interviews, we developed the content of the three workshops for pilot testing, including preparation of workshop agendas and homework between workshops. All workshops focused on managers' self-evaluation of their quality and safety work. A team of researchers and coresearchers should facilitate reflexive group discussions among the teams in the workshops. This programme was designed to take place in three workshop sessions ( 2 hours each). The content of the three pilot tested workshops was as follows:

- Workshop 1: Introduced the leadership guide and web version, identified the quality challenges that the managers experienced in their quality work, and the challenges they believed needed the most attention.

- Workshop 2: Established goals and strategies to address the identified quality challenge(s), and to involve patients/users.

- Workshop 3: Developed action plans.

Before each workshop, participants were expected to have done some preparatory reading and watched the video for that workshop (1-3). The rationale was that managers are under time constraints, and by coming prepared to the workshops, the sessions could be more time-effective and productive. For each 2-hour workshop, the research team used the detailed agenda consisting of questions, discussions, reflections and feedback sessions. The research team met between workshops to discuss the implementation and to ensure consistent approaches and reflections about findings in the two organisations in the pilot test.

\section{Phase 2: intervention pilot testing}

In the following, we present the findings from the pilot test of the intervention and how it was evaluated.

\section{The managers' use of the leadership guide}

Results from workshop observations and interviews from the pilot test showed that all managers in the nursing home and home care services were committed to and positive about using the guide and to start working with the quality challenges they identified as most relevant for their organisations.

I believe that a key part of our challenge, especially in the health service and for the nurses, is that we are so trained in suggesting specific actions and action should be implemented really fast... we are so action-oriented that we sometimes act before we have actually realized what the problem really is. So to take a step back and think about what our challenges really are is key, and then sometimes ask the 'why, why, why' questions... (Unit manager, nursing home).

In both organisations, the managers met between the workshops to collaborate on the guide, and to prepare for the next workshop. Managers in the nursing home included their employees in the quality improvement process between the workshops, as stipulated in the intervention programme. The home care organisation was undergoing reorganisation and found it more difficult to involve their employees. They needed the leadership guide to give them a sense of what quality work entails.

Moreover, results from the pilot test showed that managers in both organisations found the goals suggested in the guide too vague and difficult to operationalise. Observational data from workshops showed that managers from the home care services wanted to focus on next-of-kin involvement and a training programme for new employees, and struggled to meet one of the predefined goals. However, they appreciated the ability to use the guide to define their own goals, and consulted the predefined goals as needed. Furthermore, observation data showed that the home care managers found it important to evaluate their actions. One of their reflections during the workshop was that they were very eager to implement actions for different kinds of challenges but never followed up by evaluating them. Here, the leadership guide proved a useful evaluation tool. One of the home care managers described how the leadership guide helped them systematise their quality work:

I think it was very good at Step 1 [identification of quality challenges], that is where we haven't had any system. It clarifies. Step 3 [defining action plans] is really how we already work, we do have interventions, action plans and goals-but I see the need for the systematization in the beginning. We too often jump right into actions, and we are not so good at sorting things out and see where it belongs (...). So I think we have not applied Step 1 that much before. (Home care manager).

\section{Quality improvement tool for structure and system is important}

Results from the observations and the focus group interviews showed that the use of the guide depended on how systematically the organisations were already working on quality improvement. This also affected the extent of researcher involvement in the organisations. In the nursing home, the managers already had a well-functioning system and an established plan for their quality work, and therefore considered it a 'waste of time' to make new action plans to fit the leadership guide. Managers from both organisations insisted that working with the guide increased their awareness of their quality 
work by presenting them with new concepts and opportunities for creating new ideas, giving them a good overview of their quality work, and helping them to evaluate their actions and current practice.

(...) it has raised awareness in a way. I think. You ve got some new concept on things that you would not otherwise have had. (Unit manager, nursing home)

The results from both focus group interviews and observation showed that the managers found the seven quality challenges in the guide recognisable and informative. Managers were enthusiastic about the way in which the quality challenges gave them an overview of their organisation and quality work.

According to the managers in the pilot, the guide provided new systematics in their quality work, and they welcomed the explicit focus on patient and user-involvement. Results showed that the guide served as a checklist, and as a supplement to the quality improvement tools they already applied in their practice. In both organisations, the managers used only the web version of the guide. However, the managers stressed the need for a tutorial demonstrating its more technical capacities, such as data storage, exporting and importing of data.

\section{Manager's input on adjustments for final intervention design}

In the focus group interviews, the managers suggested that additional development of the guide and intervention should clarify that the challenges overlapped with the goals. This means that working with one challenge or goal might have a spillover effect in the guide and vice versa. Furthermore, the managers struggled to make the goals more relevant and applicable to their own organisation. Unlike the action plans, the goals are overarching and therefore not measurable, and it was important for the managers that the researchers clarify this in the final intervention programme, to prevent managers from becoming frustrated when working with the goals. In addition, the managers found that the work on goals and action plans in the guide overlapped, and experienced this as an iterative rather than a linear process. Thus, they suggested that further development of the workshops should merge the themes in workshop 2 (goals) with workshop 3 (action plans) to allow them to work with both at the same time. The nursing home managers noted that involving a user representative (a resident) in the workshops was difficult, due to the patient's reduced sight and hearing capabilities. They preferred to ask patients/users for their advice and involve them between rather than in workshops. The patients/users could then discuss their perceived challenges in the organisation and share their thoughts and experiences on more developed organisational goals and actions, as illustrated in this quote:

Yes, we should involve the patients between the workshops and get their feedback then. Then we could also approach several patients, instead of involving only one in the workshops. We could ask patients on every floor or several patients about one topic that we are concerned about. (Manager, nursing home).

\section{Phase 3: final intervention design}

Based on the results from the pilot test and discussions among project researchers and coresearchers, a final intervention programme consisting of four workshops was designed. The final intervention programme is illustrated in table 3. The following changes and adjustments from the pilot version of the intervention were made:

- Merging of the themes in workshops 2 (goals) and 3 (action plans).

- Presentation of survey results (conducted in the study prior to the intervention) to give information on quality challenges as described by the employees.

- Adding a fourth workshop on sustainability.

- Check-up call between workshops 3 and 4 .

- Development of a tutorial demonstrating the use of the web version of the leadership guide.

- Timing of intervention workshops, where workshops 1,2 and 3 should be conducted in the first 3 months of the intervention, and workshop 4 should be conducted in the sixth and final month. The rationale was to be intensively involved in the beginning so that managers would be comfortable using the guide. The fourth and last workshop could then focus on sustainability.

- The intervention programme will be implemented in the SAFE-LEAD study and evaluated by survey measurement before and after the intervention period, and process evaluation over a 1-year period as described in the study protocol. ${ }^{16}$

\section{DISCUSSION}

It is challenging to translate research-based findings into practice in healthcare settings. ${ }^{1-4} 2129$ In this paper, we have described the design of the SAFE-LEAD intervention. Our study demonstrates the importance of stakeholder involvement in the design and development of a leadership intervention in nursing homes and home care. Our approach was influenced by participatory design ${ }^{323}$ and involved coresearchers (nurse counsellors from different municipalities, patient ombudsman, patient and nextof-kin representatives), and future users (managers from nursing homes and home care). The participatory design played a key role in translating and adapting English language QUASER hospital Guide to the Norwegian nursing home and home care setting. Moreover, using this design allowed consideration of the everyday context of nursing home and home care managers, thereby tailoring the intervention content to managers' learning needs, time and challenges. In close collaboration with coresearchers and managers, the intervention was modified to fit the intended field of practice. This is consistent with previous research suggesting a more active intervention co-design involving stakeholders. ${ }^{368} 3031$

Our study supports the findings of previous research, indicating that patient and user participation in 
Table 3 Final intervention programme

\begin{tabular}{|c|c|c|c|c|}
\hline & $\begin{array}{l}\text { Workshop } 1 \\
\text { (Mnt1-2 hours) }\end{array}$ & $\begin{array}{l}\text { Workshop } 2 \\
\text { (Mnt2-2 hours) }\end{array}$ & $\begin{array}{l}\text { Workshop } 3 \\
\text { (Mnt3-2 hours) }\end{array}$ & $\begin{array}{l}\text { Workshop } 4 \\
\text { (Mnt6-2 hours) }\end{array}$ \\
\hline Preparation & $\begin{array}{l}\text { Videos and guide, } \\
\text { tutorial on web tool }\end{array}$ & Video & Video & $\begin{array}{l}\text { Check-up call before } \\
\text { work shop } 4\end{array}$ \\
\hline Introduction & $\begin{array}{l}\text { Intro of the } \\
\text { guide and web } \\
\text { tool+identify quality } \\
\text { challenges }\end{array}$ & $\begin{array}{l}\text { Status of challenges } \\
\text { and goals and reflection } \\
\text { on action plan } \\
\text { Presenting survey } \\
\text { results }\end{array}$ & $\begin{array}{l}\text { Status of challenges, } \\
\text { goals, action plans }\end{array}$ & Status \\
\hline Topic & $\begin{array}{l}\text { Seven challenges, } \\
\text { goals, user } \\
\text { involvement, action } \\
\text { plans }\end{array}$ & $\begin{array}{l}\text { Goals and action plan } \\
\text { and user involvement }\end{array}$ & Action plan & Sustainability \\
\hline Approach & $\begin{array}{l}\text { Self-diagnosis } \\
\text { facilitated by } \\
\text { researchers }\end{array}$ & $\begin{array}{l}\text { Self-diagnosis facilitated } \\
\text { by researchers }\end{array}$ & $\begin{array}{l}\text { Self-diagnosis facilitated } \\
\text { by researchers }\end{array}$ & $\begin{array}{l}\text { Self-diagnosis facilitated } \\
\text { by researchers }\end{array}$ \\
\hline Homework & $\begin{array}{l}\text { Agreement on } \\
\text { homework }\end{array}$ & $\begin{array}{l}\text { Agreement on } \\
\text { homework }\end{array}$ & $\begin{array}{l}\text { Agreement on goals for } \\
\text { ensuring sustainability }\end{array}$ & $\begin{array}{l}\text { Close involvement from } \\
\text { researchers in four units }\end{array}$ \\
\hline
\end{tabular}

The bold text specifies changes added after the pilot test.

interventions is difficult. ${ }^{30}{ }^{32}$ In our results, we attribute this to the nature of the nursing home and home care context, such as reorganisation processes, lack of managerial capabilities and the difficulty of finding patients who are physically capable of participation in projects and interventions. However, the patient and user engagement has the potential to uncover unmet needs, ${ }^{30} 32$ and hence received stronger emphasis in our adapted guide than in the original QUASER guide.

Involving coresearchers and managers in the design process generated several suggestions on how to develop a leadership intervention that would be useful and feasible in their everyday practice. They requested short meetings and suggested instructional videos and examples of when and how the guide was used by other managers, as inspiration. Therefore, we designed the intervention programme with use of blended learning activities as suggested by Harris et $a .^{22}$ Blended learning is a systematic combination of copresent (face to face) and technologically mediated interactions among students, teachers and learning resources. $^{22}$ We found that blended learning activities offered managers a more flexible way of understanding and making the best use of the leadership guide. Furthermore, the managers appreciated the fact that they saved time by watching the learning videos while preparing for a workshop and performing other tasks.

The results from the intervention design process were consistent between the managers in the nursing home and home care contexts, regarding how the leadership guide and workshops should be best designed. However, there were differences in how the two management teams used the leadership guide and adapted it to their organisation's needs and capabilities. Our study, therefore, supports earlier implementation research ${ }^{33} 34$ on the need for local adaptions in complex organisations. In line with our findings, previous research has shown that managers must be engaged in and capable of involving their management team and employees for optimal function of interventions designs. ${ }^{35-38}$ We have, therefore, designed an intervention that has a clear description of the process and that accommodates the nursing home and home care contexts and the manager's capabilities. However, despite this flexibility and adaptability, a detailed description of the intervention's elements still allows for comparison between organisations. Therefore, workshop agendas, learning resources, guide content, workshop structure are similar, but still offer choices among the seven quality challenges and goals and actions plans for their specific context.

\section{Strengths and limitations}

The main strength of this study is the thorough intervention development process that involved researchers, coresearchers, future users of the SAFE-LEAD intervention (managers in nursing homes and home care services), and patient and next-of-kin representatives, and several 


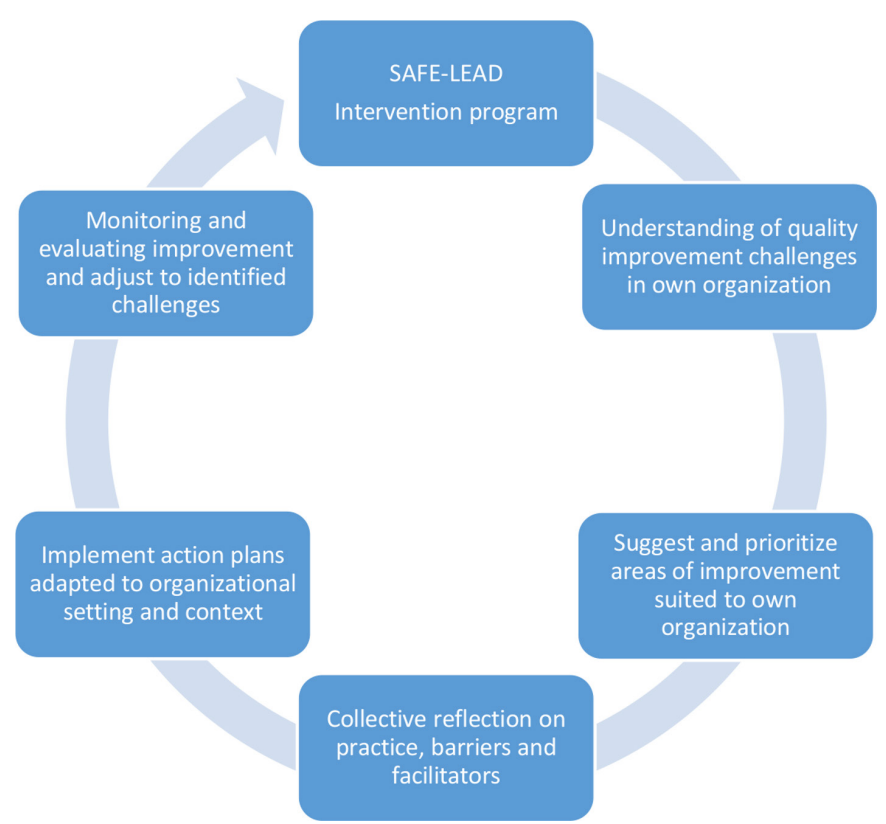

Figure 3 Logic model of the SAFE-LEAD intervention programme translating knowledge into practice based on knowledge to action framework of Straus et al. ${ }^{2}$ SAFE-LEAD, Improving Quality and Safety in Primary Care - Implementing a Leadership Intervention in Nursing Homes and Home care.

data sources (ie, focus group interviews, observations, workshops). The data material is collected from many researchers and coresearchers who contributed to the development of the intervention. Observational data are, therefore, based on subjective interpretation from many researchers.

The study has several limitations. The data material is analysed consecutively in the intervention development process, resulting in a less systematic analysis. One nursing home located in a small, rural area had only two employees participating in the focus group in the development phase, due to sick leave and understaffing. The sample of managers and units in the pilot test is limited. However, the feedback received during focus group interviews and pilot testing of the intervention was consistent across the sample, and supported by the feedback we received from the coresearchers.

There is a challenge in the intervention design on how to make it sustainable over time without researcher involvement. Quality improvement tools can be beneficial for managers in nursing homes and home care, but the heavy workload in these organisations can make implementation of improvement tools difficult without additional support in the implementation. ${ }^{39}$ Previous research shows greater chance of success with implementation if there is a stable working environment, enough personnel resources and a nursing home management that is positive to the intervention. ${ }^{40}$

In the 'Knowledge to Action' framework, Straus et $a l^{2}$ suggest several factors that should be considered when developing sustainability-oriented action plans, such as ensuring the presence of systems to monitor progress, map the availability of financial and human resources, and managers responsible for monitoring progress and ensuring sustainability. Managers can use the leadership guide to monitor progress, and no financial resources are required for using the guide. The issue of sustainability will be discussed with the participating managers during implementation of the final intervention, and a sustainability-oriented plan will be made in collaboration with researchers and managers. ${ }^{16}$ In figure 3 , we illustrate the logic model of the intervention programme and processes based on the Knowledge to Action framework. ${ }^{2}$

\section{CONCLUSION}

The findings of this study support the importance of involving stakeholders in the design of a leadership intervention. A leadership intervention for the improvement of quality and safety in nursing homes and home care contexts can be designed in a participatory approach involving stakeholders, although patient and user participation during the implementation remains a challenge. There is a need for further exploration of how patients and users can be involved in a leadership intervention and how managers can make the best use of patient and user experiences in managing quality and safety. The use of blended learning activities can be considered as a data-driven and bottom-up approach to tailor an intervention to the suggestions and educational needs identified by potential users in the development and pilot test phase. Our evaluation demonstrated that the intervention design made it easier for managers and their teams to incorporate the leadership guide into their everyday quality and safety work practice.

Acknowledgements We thank all participants in the study for sharing their knowledge and expertise with us. We also acknowledge the following members of the SAFE-LEAD Primary Care team for participating with the development of the SAFE-LEAD intervention: Karina Aase, Lene Schibevaag, Marianne Storm, Berit Ullebust (coresearcher), Marta Strandos (coresearcher), Line Hurup Thomsen (coresearcher), Elisabeth Holen-Rabbersvik (coresearcher), Mette Brevigh Nilsen, Torunn Grinvoll (coresearcher), Anne Torhild Sandvik Pedersen (coresearcher) and Elsa Kristiansen (coresearcher). We are also grateful to the reviewers for their helpful comments on our article. Language editing done by Servicescape.

Contributors All authors contributed to the research, writing and design of the manuscript. ER, SW, TS and TJ developed learning tools and collected data in workshops. IA and TJ collected data after pilot test. The data material is analysed consecutively in workshops with contribution of ER, SW, TS, TJ and IA. TJ wrote the first draft of the manuscript, while ER, SW, IA and RB critically reviewed and revised the subsequent drafts. All authors have read and approved the final manuscript.

Funding The work is part of the project Improving Quality and Safety in Primary Care-Implementing a Leadership Intervention in Nursing Homes and Home care (SAFE-LEAD Primary Care), which has received funding from the Research Council of Norway's programme HELSEVEL, under grant agreement 256681/H10, and the University of Stavanger, Norway.

Competing interests None declared.

Patient consent for publication The patient participating in the pilot test gave written informed consent.

Ethics approval The Regional Committees for Research Ethics in Norway found that the study was not regulated by the Health Research Act. The Norwegian Centre for Research Data approved the study in two phases (phase 1: NSD, ID 52324; phase 2: NSD, ID 54855). The study followed the Declaration of Helsinki. 
Provenance and peer review Not commissioned; externally peer reviewed.

Data sharing statement Anonymised data of the study will be stored at the Norwegian Social Science Data Services subsequent to completion of the project, and will then be available to others on request.

Open access This is an open access article distributed in accordance with the Creative Commons Attribution Non Commercial (CC BY-NC 4.0) license, which permits others to distribute, remix, adapt, build upon this work non-commercially, and license their derivative works on different terms, provided the original work is properly cited, appropriate credit is given, any changes made indicated, and the use is non-commercial. See: http://creativecommons.org/licenses/by-nc/4.0/.

\section{REFERENCES}

1. Tsoukas $\mathrm{H}$. The firm as a distributed knowledge system: $\mathrm{A}$ constructionist approach. Strategic Management Journal 1996;17(S2):11-25.

2. Straus S, Tetroe J, Graham ID. Knowledge translation in health care: moving from evidence to practice. 2 ed. Wiley Blackwell: Oxford, 2013.

3. Marshall M, Pagel C, French C, et al. Moving improvement research closer to practice: the Researcher-in-Residence model. BMJ Qual Saf 2014;23.

4. Marshall M, Mountford J. Developing a science of improvement. J R Soc Med 2013;106:45-50.

5. Øvretveit J. Understanding the conditions for improvement: research to discover which context influences affect improvement success. BMJ Qual Saf 2011;20 Suppl 1(Suppl 1):i18-i23.

6. Marshall M, Silva de D, Cruickshank L. What we know about designing and effective improvement intervention (but too often fail to put into practice). BMJ 2017;26:578-82.

7. McDonald KM. Considering context in quality improvement interventions and implementation: concepts, frameworks, and application. Acad Pediatr 2013;13:S45-S53.

8. Ward ME, De Brún A, Beirne D, et al. Using Co-Design to Develop a Collective Leadership Intervention for Healthcare Teams to Improve Safety Culture. Int J Environ Res Public Health 2018;15:1182.

9. Vogelsmeier A, Scott-Cawiezell J. Achieving quality improvement in the nursing home: influence of nursing leadership on communication and teamwork. J Nurs Care Qual 2011;26:236-42.

10. Parand A, Dopson S, Renz A, et al. The role of hospital managers in quality and patient safety: a systematic review. BMJ Open 2014;4:e005055.

11. Künzle B, Kolbe M, Grote G. Ensuring patient safety through effective leadership behaviour: A literature review. Saf Sci 2010;48:1-17.

12. Jones L, Pomeroy L, Robert G, et al. Explaining organisational responses to a board-level quality improvement intervention: findings from an evaluation in six providers in the English National Health Service. BMJ Qual Saf 2019;28:198-204.

13. Jones L, Pomeroy L, Robert G, et al. How do hospital boards govern for quality improvement? A mixed methods study of 15 organisations in England. BMJ Qual Saf 2017;26:978-86.

14. Bresnen M, Hodgson D, Bailey S, et al. Mobilizing management knowledge in healthcare: Institutional imperatives and professional and organizational mediating effects. Manag Learn 2017;48:597-614.

15. Vaughn VM, Saint S, Krein SL, et al. Characteristics of healthcare organisations struggling to improve quality: results from a systematic review of qualitative studies. BMJ Qual Saf 2019;28:74-84.

16. Wiig S, Ree E, Johannessen $\mathrm{T}$, et al. Improving quality and safety in nursing homes and home care: the study protocol of a mixedmethods research design to implement a leadership intervention. BMJ Open 2018;8:e020933.
17. Ministry of Health and Care Services. Meld St. 10 (2012-2013). God kvalitet - trygge tjenester - Kvalitet og pasientsikkerhet i helse- og omsorgstjenesten. Oslo, 2012.

18. Ministry of Health and Care Services. Meld St. 11 (2014-2015). Kvalitet og pasientsikkerhet 2013. Oslo, 2014.

19. Quaser FN. The Hospital Guide. A research-based tool to reflect on and develop your quality improvement strategies: University College London. 2013 https://www.ucl.ac.uk/dahr/pdf/study_documents/ iQUASER_Hospital_Guide_291014_press-ready_cs4.pdf (cited 17 Nov 2017)

20. Bate P, Mendel P, Robert G. Organizing for quality: the improvement journeys of leading hospitals in Europe and the United States: Radcliffe Publishing, 2008.

21. Craig P, Dieppe P, Macintyre S, et al. Developing and evaluating complex interventions: the new Medical Research Council guidance. BMJ 2008;337:a1655.

22. Harris P, Connolly J, Feeney L. Blended learning: overview and recommendations for successful implementation. Industrial and Commercial Training 2009;41:155-63.

23. Bate P, Robert G. Bringing User Experience to Healthcare Improvement: Radcliffe Publishing Oxford, 2007.

24. Thabane L, Ma J, Chu R, et al. A tutorial on pilot studies: the what, why and how. BMC Med Res Methodol 2010;10:1.

25. Strøm A, Fagermoen MS. Systematic Data Integration-A Method for Combined Analyses of Field Notes and Interview Texts. International Journal of Qualitative Methods 2012;11:534-46.

26. Hsieh HF, Shannon SE. Three approaches to qualitative content analysis. Qual Health Res 2005;15:1277-88.

27. Patton M. Qualitative research and evaluation methods. 3rd ed. Thousand Oaks: Sage, 2002.

28. Fangen K. Deltakende observasjon [Participant observation. $2^{\text {nd }}$ ed. Bergen: Fagbokforlaget, 2010.

29. Dixon-Woods M, McNicol S, Martin G. Ten challenges in improving quality in healthcare: lessons from the Health Foundation's programme evaluations and relevant literature. BMJ Qual Saf 2012;21:876-84.

30. Owens C, Farrand P, Darvill R, et al. Involving service users in intervention design: a participatory approach to developing a textmessaging intervention to reduce repetition of self-harm. Health Expect 2011;14:285-95.

31. Staniszewska S, Jones $\mathrm{N}$, Newburn $\mathrm{M}$, et al. User involvement in the development of a research bid: barriers, enablers and impacts. Health Expect 2007;10:173-83.

32. Bridgelal Ram M, Grocott PR, Weir HC. Issues and challenges of involving users in medical device development. Health Expect 2008;11:63-71.

33. Greenhalgh T, Robert G, Macfarlane F, et al. Diffusion of innovations in service organizations: systematic review and recommendations. Milbank Q 2004;82:581-629.

34. May C. Agency and implementation: understanding the embedding of healthcare innovations in practice. Soc Sci Med 2013;78:26-33.

35. Oldenhof L, Postma J, Putters K. On justification work: how compromising enables public managers to deal with conflicting values. Public Adm Rev 2014;74:52-63.

36. Oldenhof L, Stoopendaal A, Putters K. From boundaries to boundary work: middle managers creating inter-organizational change. $J$ Health Organ Manag 2016;30:1204-20.

37. Clegg SR, Kornberger M, Rhodes C. Learning/becoming/organizing. Organization 2005;12:147-67.

38. Birken SA, Lee SY, Weiner BJ. Uncovering middle managers'role in healthcare innovation implementation. Implement Sci 2012;7:28-9.

39. Jiwa M, McManus A, Dadich A, et al. Harnessing information technology to innovate in primary care. Qual Prim Care 2013;21:43-9.

40. Malterud K, Aamland A, Iden KR. Small-scale implementation with pragmatic process evaluation: a model developed in primary health care. BMC Fam Pract 2018;19:93. 